\title{
Hyperlipidemia due to Nephrotic Syndrome: Its Effects and Effects of Interventions on Atherogenesis, Cardiovascular and Renal Outcomes
}

\author{
Hidekatsu Yanai
}

\begin{abstract}
Nephrotic syndrome (NS) is one of the most important causes of secondary hyperlipidemia. Here, I describe characteristics and mechanisms for hyperlipidemia due to NS, and systematically reviewed the association of such hyperlipidemia with atherosclerotic progression and the development of cardiovascular diseases (CVD) by Pubmed. Further, I searched literatures on the effects of interventions including diet, statin, fibrates, low-density lipoprotein (LDL)-apheresis and proprotein convertase subtilisin/kexin type 9 (PCSK9) inhibitors on hyperlipidemia and cardiovascular and renal outcomes in NS patients. Although dyslipidemia may be associated with atherosclerosis in NS, other factors such as age, duration of disease, number of relapses and blood pressure are also crucial determinants of atherosclerosis. The disease-specific risk of thromboembolism was different across the histological groups. One cohort study suggested that persons with NS are at increased risk of coronary heart disease (CHD). Among various interventions for NS, statin is relatively safe and effective for hyperlipidemia due to NS, but, it does not show sufficient evidence for cardiovascular and renal outcomes. Although PCSK9 inhibitors are promising therapeutic options for NS, large-scale trials are needed to elucidate such effect.
\end{abstract}

Keywords: Hyperlipidemia; Nephrotic syndrome; Cardiovascular disease; Lipoprotein; Coronary heart disease

\section{Introduction}

Nephrotic syndrome (NS) is one of the most common kidney diseases in children and adults, and is characterized by massive proteinuria, edema and hypoalbuminemia [1]. Although the majority of patients respond to initial treatment with glucocorticoids by entering into clinical remission, a substantial

Manuscript submitted June 29, 2020, accepted July 10, 2020

Published online August 26, 2020

Department of Diabetes, Endocrinology and Metabolism, National Center for Global Health and Medicine Kohnodai Hospital, 1-7-1 Kohnodai, Ichikawa, Chiba 272-8516, Japan. Email: dyanai@hospk.ncgm.go.jp

doi: https://doi.org/10.14740/jem663 proportion of patients ( $20 \%$ of children and $50 \%$ of adults) either present with or subsequently develop clinical steroid resistance during the course of their disease $[2,3]$. Various complications such as infection, acute kidney injury and thromboembolism may develop due to persistence of the nephrotic state and/or from exposure to the relatively toxic alternative therapies that are used to induce remission [4]. Since patients with NS include a wide range of age and various histological groups such as minimal change disease, immunoglobin A (IgA) nephropathy, membranous nephropathy (MN) and membranoproliferative glomerulonephritis, it is difficult to pick up patients studied to elucidate its involvement in atherosclerosis.

\section{Dyslipidemia in NS}

The major lipoproteins, including intermediate-density lipoprotein (IDL), very low-density lipoprotein (VLDL) and lowdensity lipoprotein (LDL) are increased in plasma of patients with NS, therefore, patients with NS show elevated serum cholesterol and triglyceride (TG) levels [4]. Such dyslipidemia is owing mainly to impaired clearance and, to a lesser extent, increased biosynthesis. Impaired clearance is a direct result of decreased hepatic lipase activity and decreased lipoprotein lipase (LPL) activity in the endothelium and peripheral tissues [4]. In addition, hepatic levels of proprotein convertase subtilisin/kexin type 9 (PCSK9) which degrades the LDL receptor are increased in patients with NS [4], inducing further elevation of LDL.

\section{NS and Atherosclerosis and Cardiovascular Diseases (CVDs)}

In general, dyslipidemia accelerates atherosclerosis, and increases risk of myocardial infarction (MI) and cerebrovascular accident. Collected literatures on NS and the makers for atherosclerosis and cardiovascular events were shown in Table 1 $[5,6,9-14]$.

\section{NS and the makers for atherosclerosis}

A case control study was conducted in 66 children with NS. 

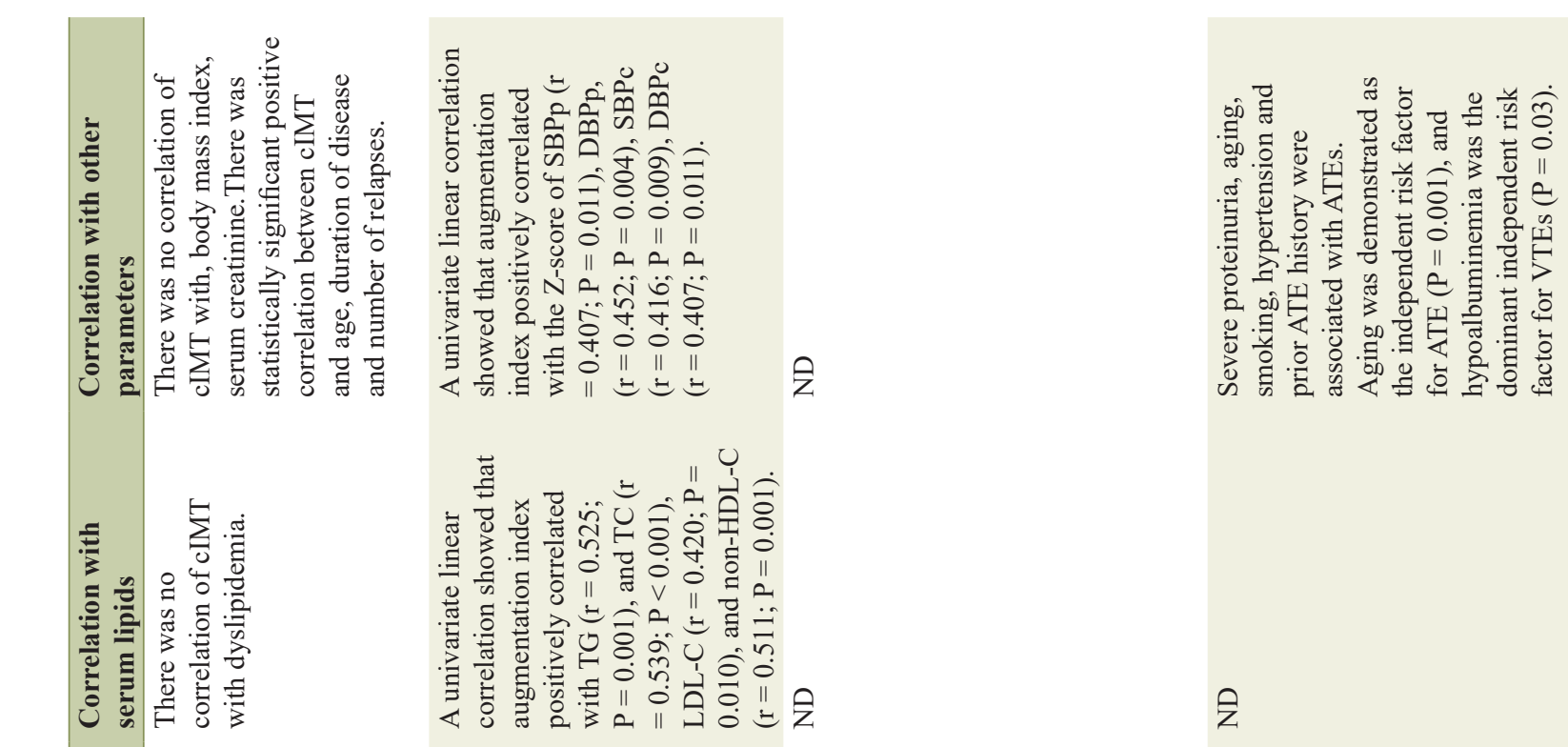

ส $\gtrsim$

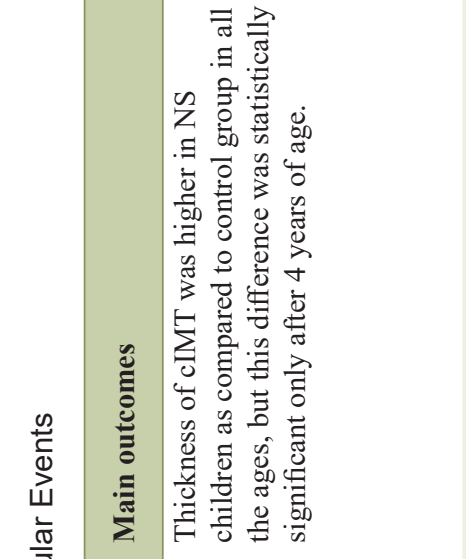

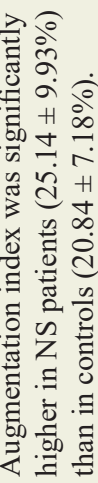

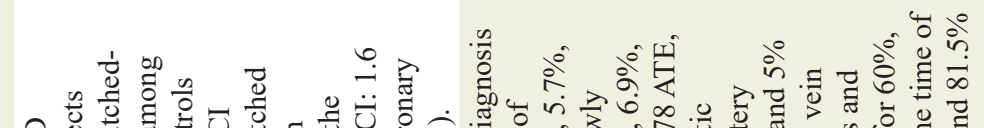

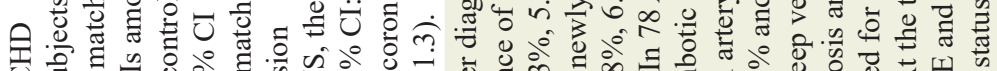

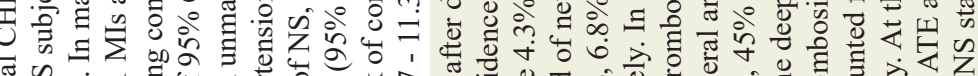

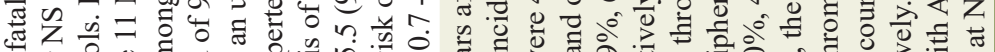

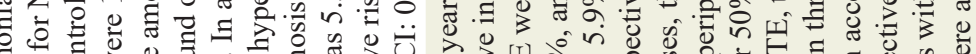

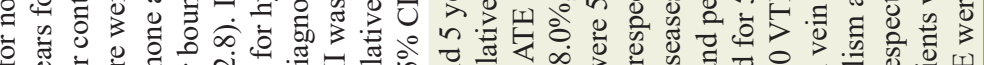
过

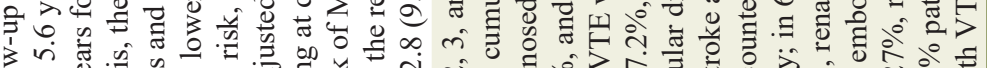

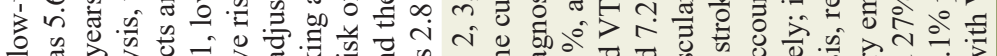

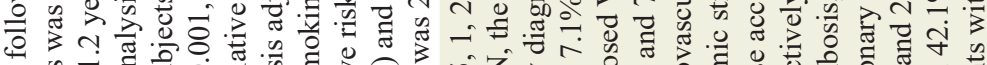

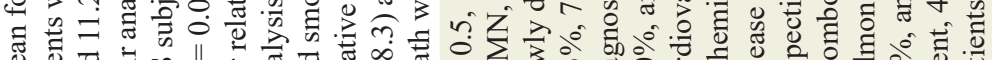

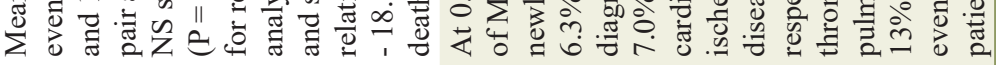

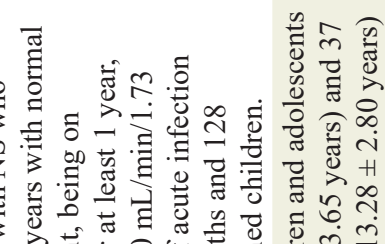

वृत

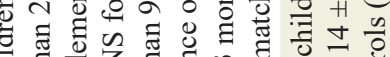

可

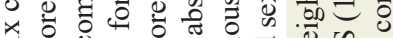

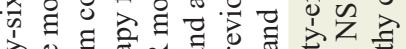

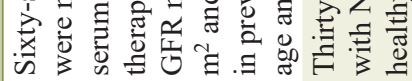

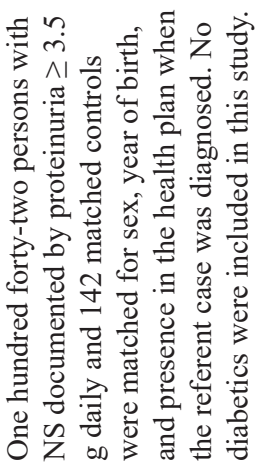

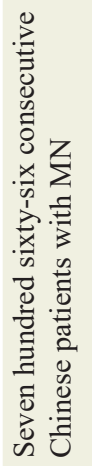

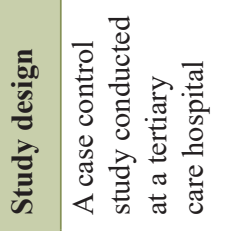

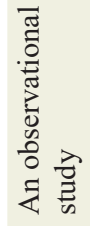

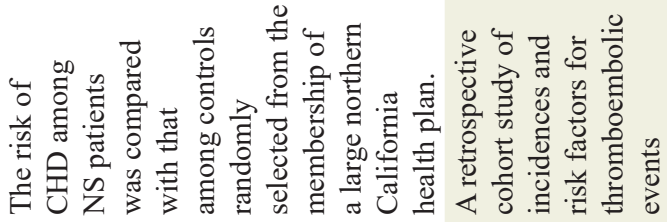

里

$\frac{\dot{0}}{\frac{0}{0}}$

तो สं

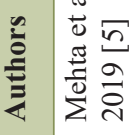

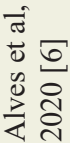

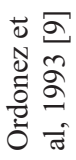

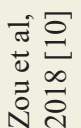




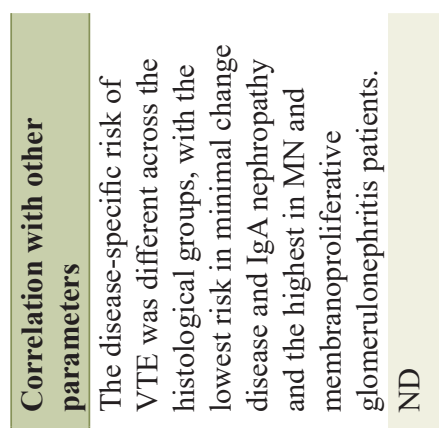

z

令
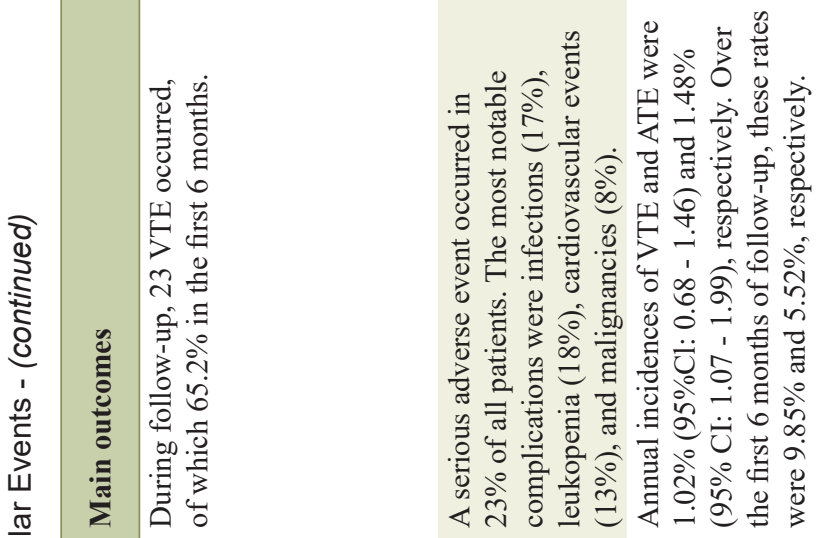

चึ ڤ્

点䓠

त)

‡.

हें

응 है

o.

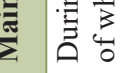

苞

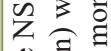

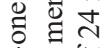

ฮ

氙密

to

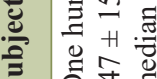

苍吾导导

cond

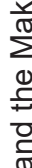

$\frac{f}{\mathrm{D}}$

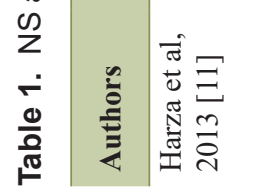
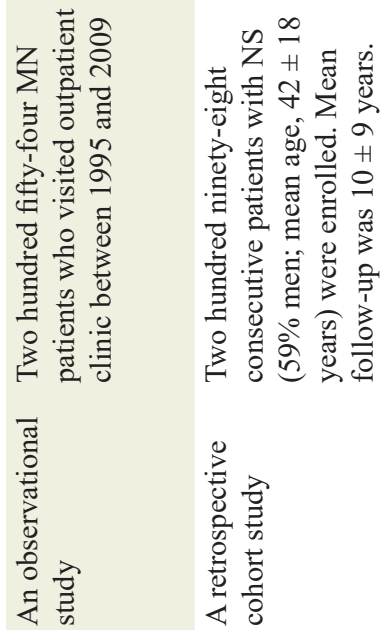
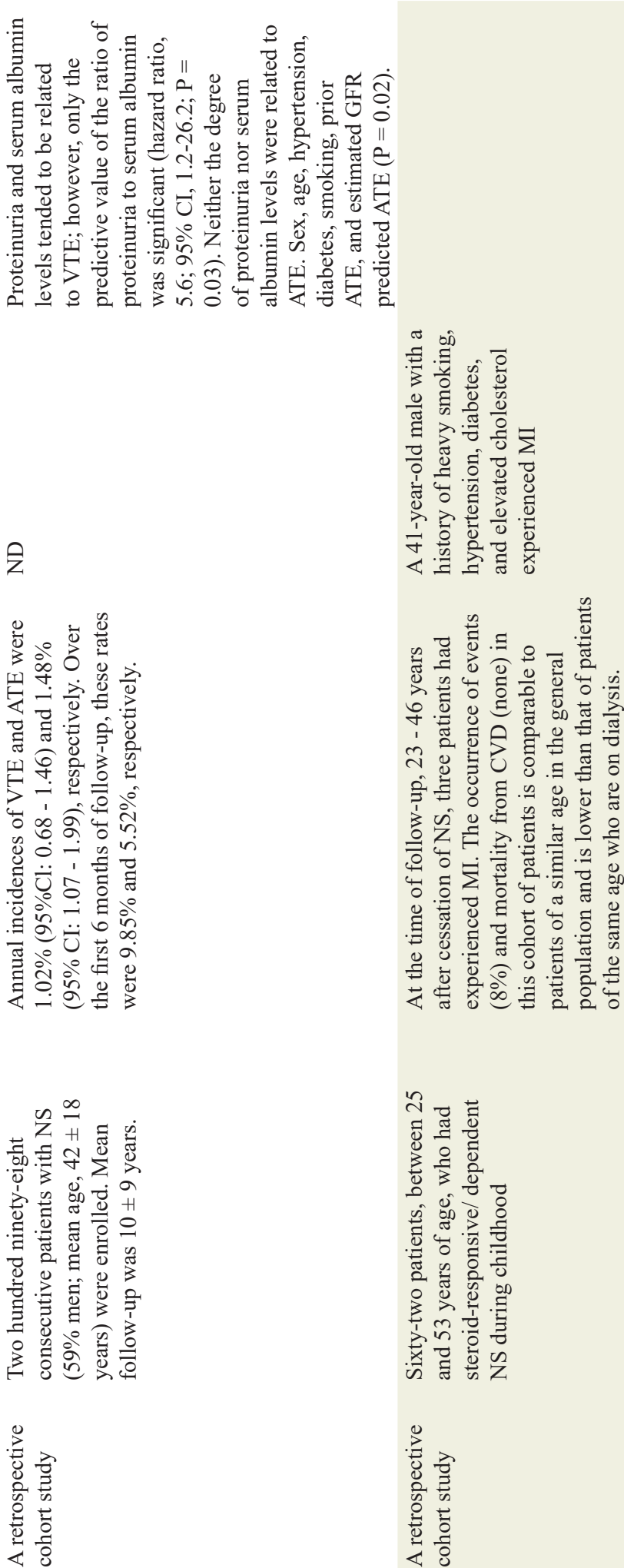

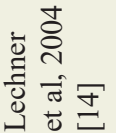

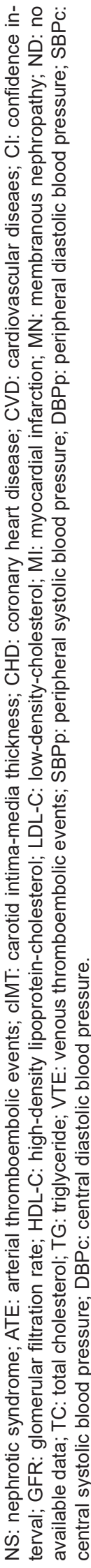


Thickness of carotid intima-media thickness (cIMT) was higher in NS children as compared to control group in all the ages, but this difference was statistically significant only after 4 years of age [5]. There was statistically significant positive correlation between cIMT and age, duration of disease and number of relapses. There was no correlation of cIMT with dyslipidemia. In another observational study of 38 children and adolescents with NS and 37 healthy controls, augmentation index as the marker for arteriosclerosis was significantly higher in NS patients than in controls [6]. A univariate linear correlation showed that augmentation index positively correlated with TG $(\mathrm{r}=0.525 ; \mathrm{P}=0.001)$, and total cholesterol $(\mathrm{TC})$ $(\mathrm{r}=0.539 ; \mathrm{P}<0.001)$, LDL-cholesterol (C) $(\mathrm{r}=0.420 ; \mathrm{P}=$ $0.010)$, and non-high-density lipoprotein (HDL)-C ( $\mathrm{r}=0.511$; $\mathrm{P}=0.001$ ) [6]. A univariate linear correlation showed that augmentation index also positively correlated with the Z-score of central and peripheral blood pressure.

Although dyslipidemia may be associated with atherosclerosis in NS, other factors such as age, duration of disease, number of relapses and blood pressure are also crucial determinants of atherosclerosis.

\section{NS and CVD}

Children with NS develop thromboembolism at a rate of $2.8 \%$, whereas adults have a much higher rate of $26.7 \%$ [7]. Thromboembolism may be due to increased urinary loss of antithrombotic factors and increased hepatic production of prothrombotic factors $[7,8]$.

The risk of coronary heart disease (CHD) among 142 persons with NS was compared with that among 142 matched controls randomly selected from the membership of a large northern California health plan [9]. In matched-pair analysis, there were 11 cases with MI among NS subjects and none among controls $(\mathrm{P}=0.001)$. In an unmatched analysis adjusted for hypertension and smoking at diagnosis of $\mathrm{NS}$, the relative risk of MI was 5.5 (95\% confidence interval (CI): $1.6-18.3)$ and the relative risk of coronary death was 2.8 (95\% CI: 0.7 - 11.3), suggesting that persons with NS are at increased risk of CHD.

A cohort study of incidences and risk factors for thromboembolic events in 766 consecutive Chinese patients with MN showed that at $0.5,1,2,3$, and 5 years after diagnosis, the cumulative incidence of newly diagnosed arterial thromboembolic events (ATE) were 4.3\%, 5.7\%, 6.3\%, 7.1\%, and $8.0 \%$, respectively [10]. In 78 ATE, CVD, thrombotic ischemic stroke and peripheral artery disease accounted for $50 \%, 45 \%$ and $5 \%$ respectively. At the time of event, $42.1 \%$ patients with ATE were at NS status, suggesting that NS is the crucial risk factor for ATE. Severe proteinuria, aging, smoking, hypertension and prior ATE history were associated with ATEs. A prospective observational study including 191 adult patients with NS showed that during follow-up of 24 months, 23 venous thromboembolic events (VTE) occurred, of which $65.2 \%$ in the first 6 months [11]. The disease-specific risk of VTE was different across the histological groups, with the lowest risk in minimal change disease and $\operatorname{IgA}$ nephropathy and the highest in $\mathrm{MN}$ and membranoproliferative glomerulonephri- tis patients [11]. In $254 \mathrm{MN}$ patients who visited outpatient clinic between 1995 and 2009, cardiovascular events occurred in $13 \%$ of MN patients [12]. Retrospective cohort study of 298 consecutive patients with NS showed that annual incidences of VTE and ATE were $1.02 \%$ and $1.48 \%$, respectively [13]. Over the first 6 months of follow-up, these rates were $9.85 \%$ and $5.52 \%$, respectively. Proteinuria and serum albumin levels tended to be related to VTE; however, only the predictive value of the ratio of proteinuria to serum albumin was significant [13]. Sex, age, hypertension, diabetes, smoking, prior ATE, and estimated glomerular filtration rate (GFR) predicted ATE $(P=0.02)$. Lechner et al reported that the occurrence of CVD events $(8 \%)$ and mortality from CVD (none) in the cohort of steroid-responsive NS during childhood patients is comparable to patients of a similar age in the general population [14], suggesting that steroid responsive NS is unlikely to develop CVD.

\section{Outcomes of Interventions on Dyslipidemia in NS}

\section{Diet}

Dietary interventions on NS were shown in Table 2 [15-17]. Twenty untreated patients with severe proteinuria and hyperlipidemia were instructed to eat a vegetarian soy diet for 8 weeks [15]. The diet was low in fat and protein (daily $0.71 \mathrm{~g} /$ $\mathrm{kg}$ ideal body weight), cholesterol free, and rich in monounsaturated and polyunsaturated fatty acids and in fiber. During the soy-diet period, there were significant decrease in serum TC, LDL-C, and HDL-C and apolipoproteins (apos) A and $\mathrm{B}$, but serum $\mathrm{TG}$ did not change. Urinary protein decreased significantly. Serum lipids and proteinuria tended to return towards baseline values during the washout period. Twenty patients with NS and hyperlipidemia were randomly allocated either on soy diet alone or to soy diet plus $5 \mathrm{~g}$ /day of fish oil for 2 months [16]. Then they crossed over to the other treatment for two additional months. With the soy diet, a significant decrease of hyperlipidemia and proteinuria were observed. The effect of the soy diet on proteinuria increased over the 4 months. The addition of fish oil had no further beneficial effect. Fasting lipids and lipoproteins in 17 patients with nephrotic range proteinuria and 17 age and sex matched controls were measured before and after 8 weeks treatment with $4 \mathrm{~g}$ daily of omega-3 fatty acids [17]. The omega-3 fatty acids treatment significantly reduced TG and VLDL-C, small dense LDL, remnant-like particles (RLP)-C and RLP-TG. However, insignificant rise of LDL-C was observed. Omega-3 fatty acids intake did not alter HDL-C.

\section{Statin}

Effects of Statin on NS were shown in Table 3 [18-24]. The efficacy, safety, and tolerability of simvastatin (20 mg twice a day) in the treatment of hyperlipidemia due to unremitting NS was compared with that of cholestyramine ( $8 \mathrm{~g}$ twice a day) in a crossover trial [18]. Simvastatin was significantly more 


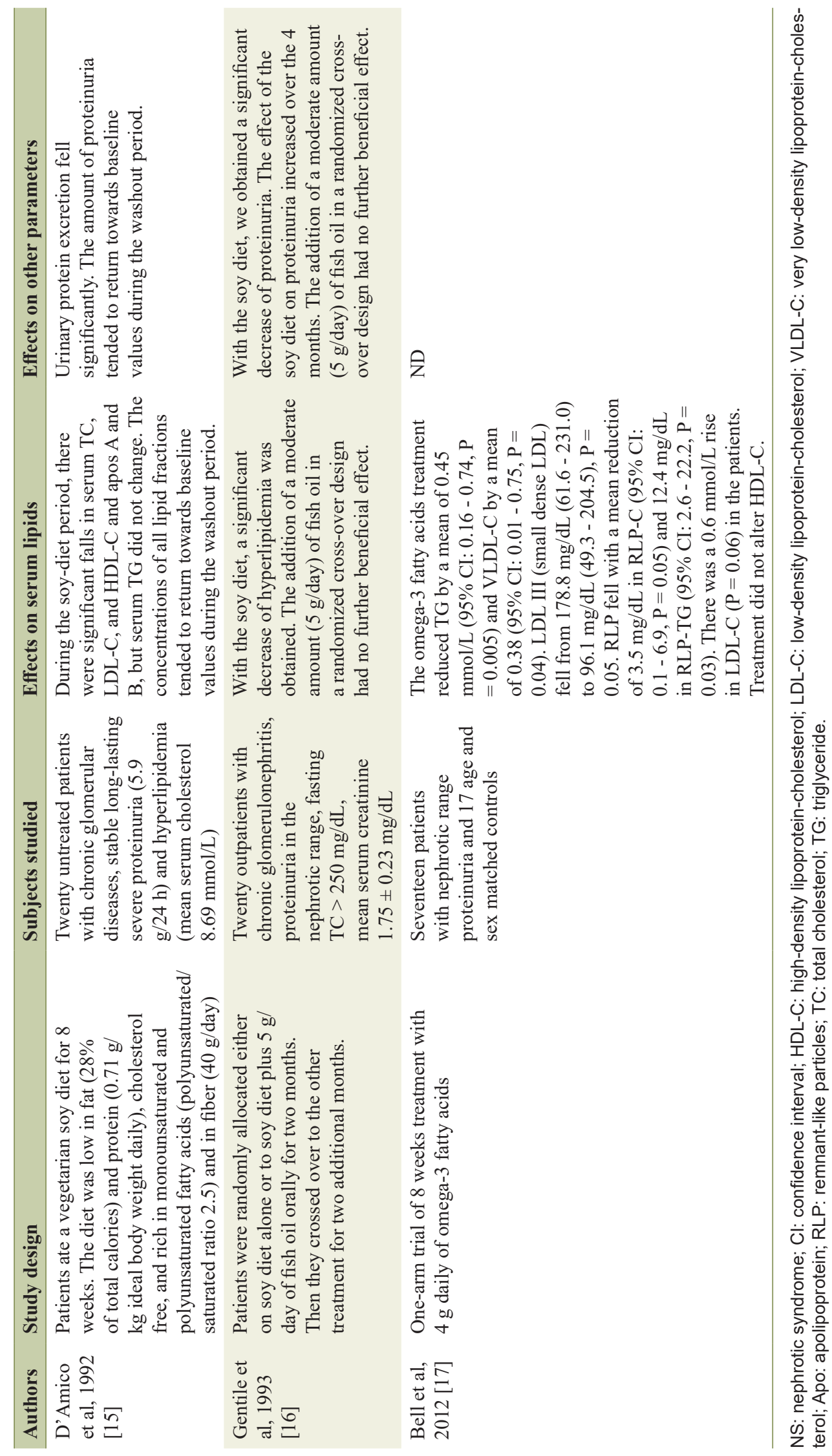




\begin{tabular}{|c|c|c|c|c|c|c|}
\hline & 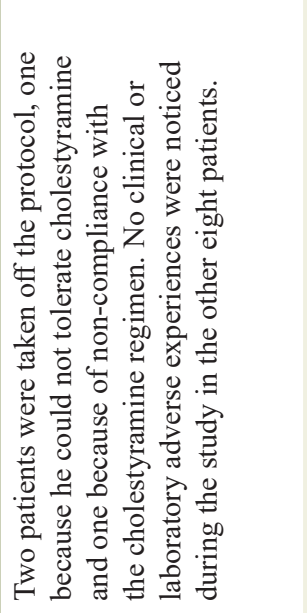 & 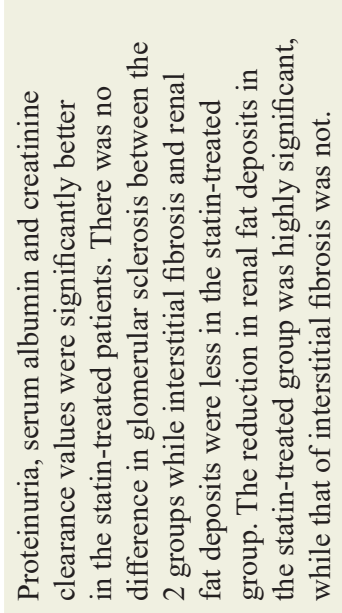 & 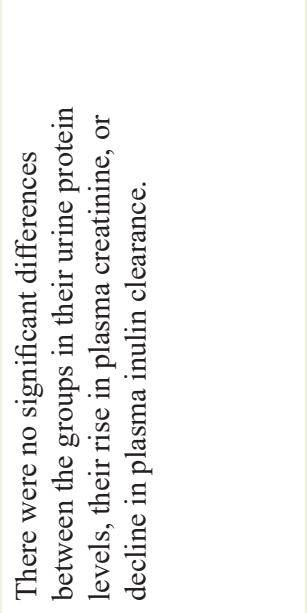 & 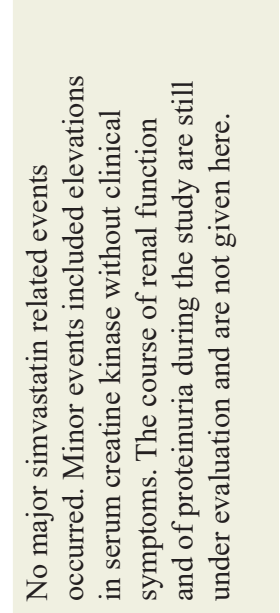 & 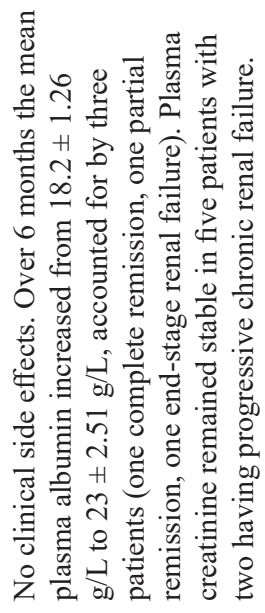 & 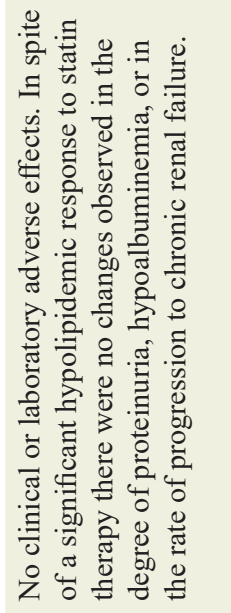 \\
\hline & 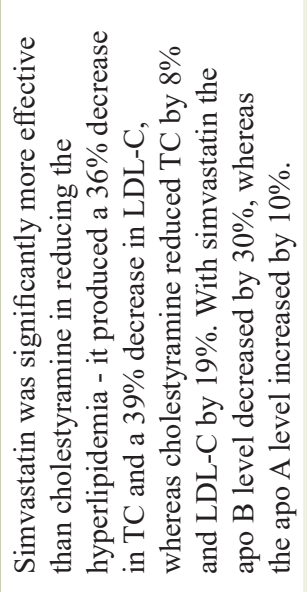 & 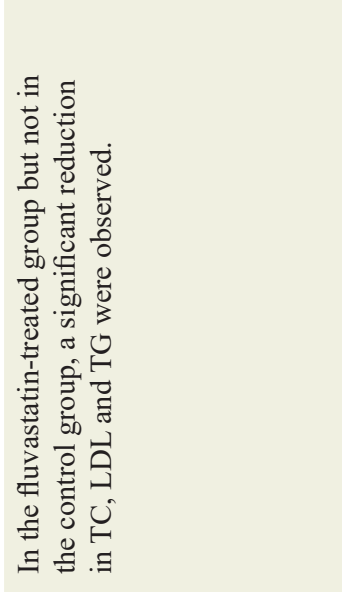 & 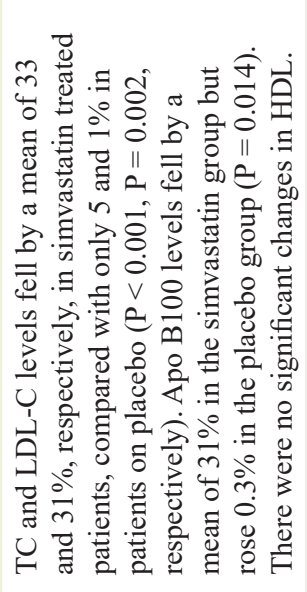 & 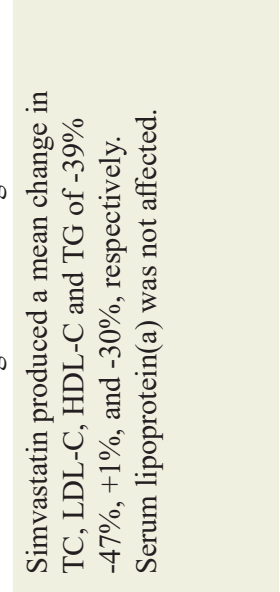 & 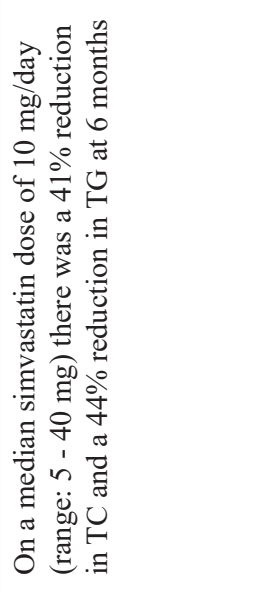 & 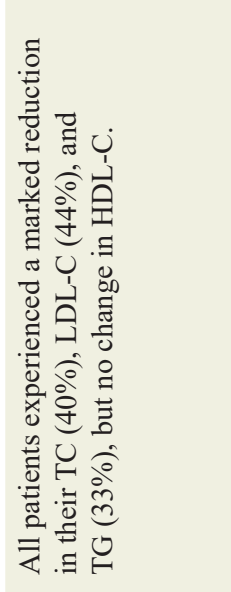 \\
\hline & 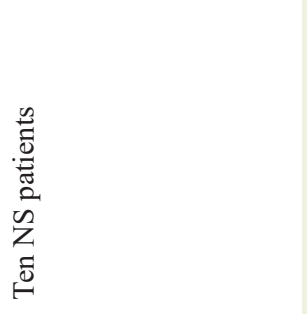 & 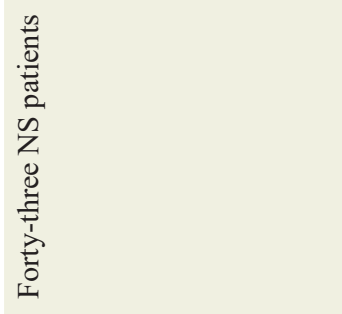 & 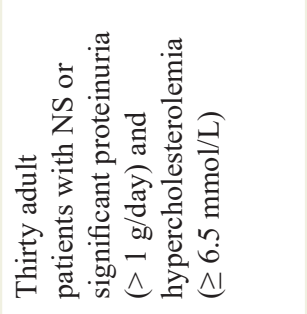 & 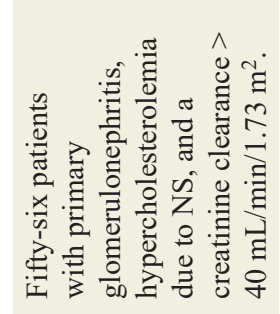 & 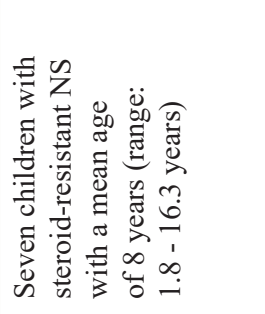 & 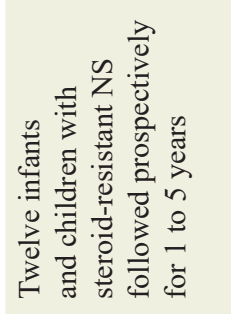 \\
\hline & 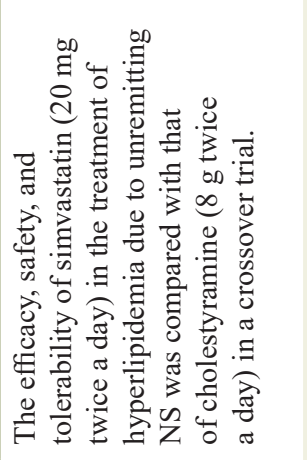 & 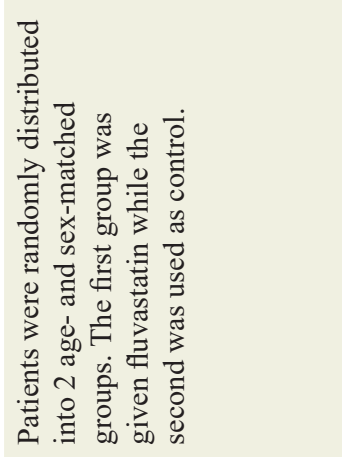 & 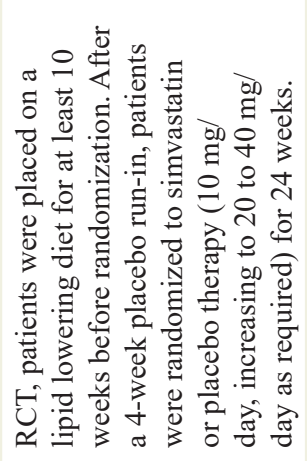 & 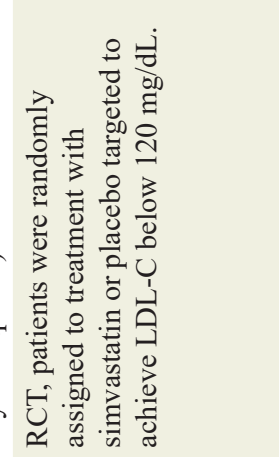 & 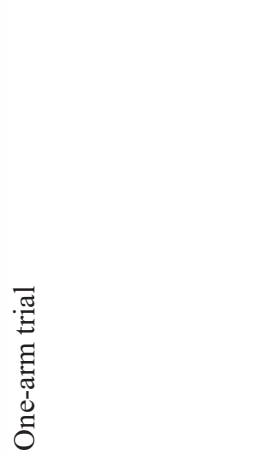 & 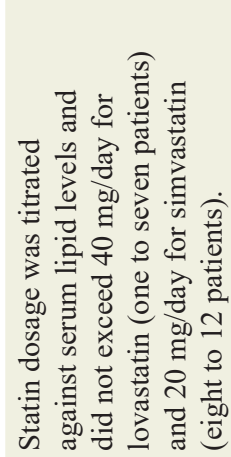 \\
\hline & 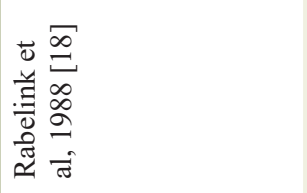 & 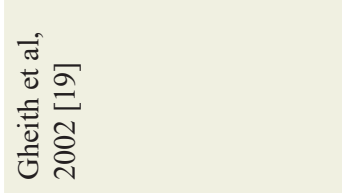 & 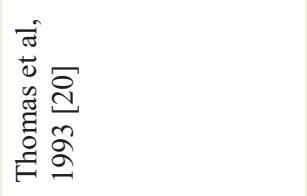 & 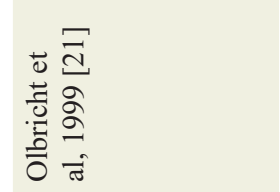 & 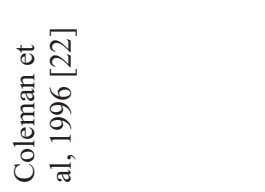 & 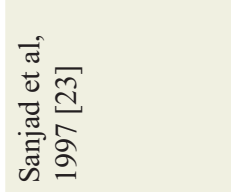 \\
\hline
\end{tabular}


effective than cholestyramine in reducing TC, LDL-C and apo B. Forty-three NS patients were randomly distributed into two age- and sex-matched groups (fluvastatin vs. control) [19]. In the fluvastatin-treated group but not in the control group, a significant reduction in TC, LDL and TG were observed. Proteinuria, serum albumin and creatinine clearance values were significantly better in the statin-treated patients [19]. Interstitial fibrosis and renal fat deposits were less in the statintreated group. Thirty patients were placed on a lipid lowering diet for at least 10 weeks before randomization, and after a 4-week placebo run-in, patients were randomized to simvastatin or placebo therapy $(10 \mathrm{mg} /$ day, increasing to 20 to 40 $\mathrm{mg}$ /day as required) for 24 weeks [20]. TC and LDL-C fell by a mean of 33 and $31 \%$, respectively, in simvastatin treated patients, an apo B100 fell by a mean of $31 \%$. There were no significant differences between the groups in their urine protein levels, their rise in plasma creatinine, or decline in plasma inulin clearance. Fifty-six NS patients were randomly assigned to treatment with simvastatin or placebo targeted to achieve LDL-C below $120 \mathrm{mg} / \mathrm{dL}$ [21]. Simvastatin produced a mean change in TC, LDL-C, HDL-C and TG of $-39 \%-47 \%,+1 \%$, and $-30 \%$, respectively. Seven children with steroid-resistant NS took simvastatin dose of $10 \mathrm{mg} /$ day (range: 5 - $40 \mathrm{mg}$ ) [22]. There was a $41 \%$ reduction in $\mathrm{TC}$ and a $44 \%$ reduction in TG at 6 months. Over 6 months the mean plasma albumin concentrations increased. Plasma creatinine concentrations remained stable in five patients. Twelve infants and children with steroid-resistant NS took lovastatin or simvastatin [23]. All patients experienced a hypolipidemic response with a marked reduction in TC (40\%), LDL-C (44\%), and TG (33\%), but no change in HDL-C. There were no changes observed in the degree of proteinuria, hypoalbuminemia, or in the rate of progression to chronic renal failure. Thirty patients received a fixed dose of atorvastatin $(\mathrm{n}=15,10 \mathrm{mg} /$ day $)$ or placebo $(\mathrm{n}=$ 15) by block randomization in a $1: 1$ ratio [24]. At the end of 12 months, atorvastatin was not superior to placebo in reducing plasma LDL-C levels. Apo B levels significantly declined with atorvastatin in modified intention-to-treat analysis $(\mathrm{P}=$ 0.01 ) but not in the per-protocol analysis. There was no significant effect on other lipid fractions, cIMT and flow-mediated dilation. Change in serum albumin was negatively associated with change in serum LDL-C, VLDL-C, TC, TG, and apo B $(\mathrm{P}<0.001)$, irrespective of receiving atorvastatin, age, gender, body mass index, and serum creatinine.

Most of studies showed that statin use is associated with reduction in TC, LDL-C, and TG. Out of seven studies, five studies reported renal outcome. Two studies showed an improvement of proteinuria due to statin, however, three studies did not show any beneficial effects of statin on renal outcome.

\section{Fibrate}

Effects of fibrate on NS were shown in Table 4 [25, 26]. Gemfibrozil $600 \mathrm{mg}$ or placebo was administered to $11 \mathrm{NS}$ patients twice a day with 6-week treatment periods [25]. There was a third unblinded period in which seven patients received gemfibrozil plus the bile acid-binding resin, colestipol, $10 \mathrm{~g}$ twice a day. Gemfibrozil treatment produced a marked reduction in 


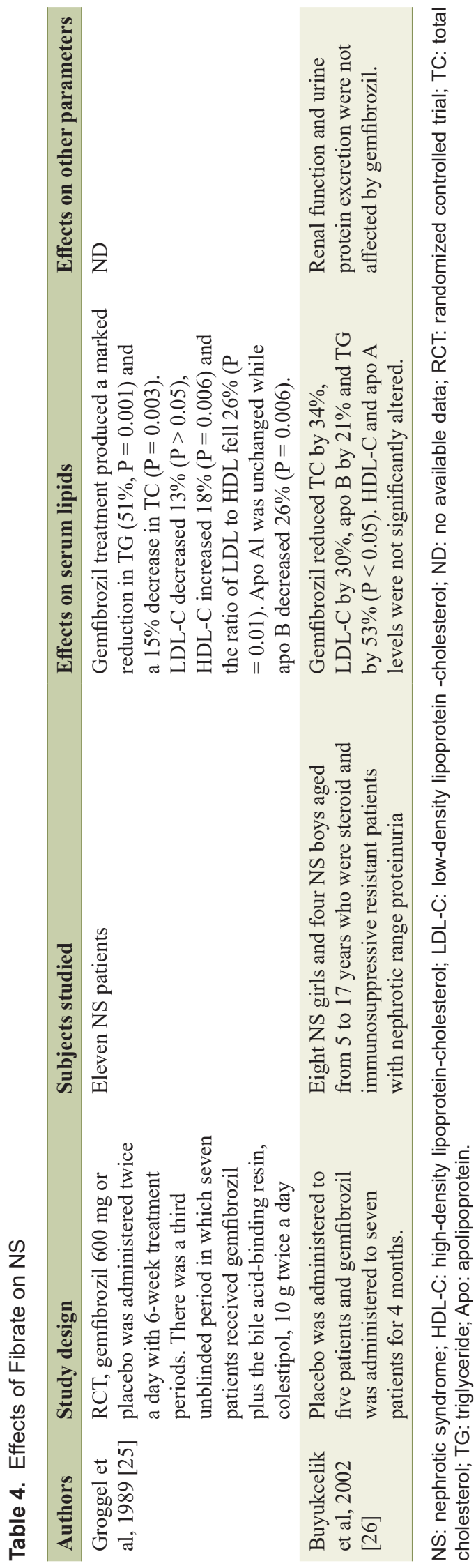

TG and TC. LDL-C significantly decreased, and HDL-C significantly increased, and a significant decrease of the ratio of LDL to HDL was also obtained. Apo A1 was unchanged while apo B decreased. Renal outcome due to gemfibrozil was not reported. Placebo was administered to five patients and gemfibrozil was administered to seven patients for 4 months [26]. Gemfibrozil significantly reduced TC, LDL-C, apo B and TG. HDL-C and apo A levels were not significantly altered. Renal function and urine protein excretion were not affected by gemfibrozil.

Pemafibrate, a novel selective peroxisome proliferatoractivated receptor $\alpha$ modulator (SPPARM $\alpha$ ), showed the superiority of pemafibrate on effects on serum TG reduction and HDL-C elevation as well safety [27]. Although previous fibrates showed worsening of kidney function test values, pemafibrate was less likely to increase serum creatinine or decrease eGFR [27]. However, the interventional trial for NS using pemafibrate has not been performed yet, which is desired in the future.

\section{LDL-apheresis (LDL-A)}

Effects of LDL-A on NS were shown in Table 5 [28-31]. LDL-A was performed twice a week for 3 weeks (first course), then weekly for 6 weeks (second course) [28]. Eleven patients who had biopsy-proven focal segmental glomerulosclerosis (FGS) presenting with NS and were resistant to steroid and conventional-dose cyclosporine A therapy were included. Beginning from the second LDL-A course, a dosage of $1 \mathrm{mg} / \mathrm{kg} /$ day of prednisone was administered for 6 weeks, then tapered. Serum TC and TG significantly decreased. Seven patients experienced remission of NS, five of whom achieved complete remission within 4 weeks after initiating prednisone therapy with LDL-A [28]. Complete remission was associated with significantly more highly selective proteinuria compared with treatment failure [28]. In another study investigating the effects of combined therapy LDL-A and steroid in FGS patients, a significant decrease of urinary protein and increase of serum albumin were obtained by this treatment [29]. The rate of entering complete or incomplete remission was $71 \%$ with a relatively short duration of nephrotic-range proteinuria using the LDL-A therapy in comparison with steroid therapy alone.

In 44 patients with drug-resistant NS, along with rapid improvement of hyperlipidemia, LDL-A significantly improved proteinuria and hypoproteinemia [30]. More than half of the patients showed remission of NS based on the urinary protein level at the completion of LDL-A. In 58 refractory NS patients from 40 facilities, 21 patients $(47.7 \%)$ showed remission of NS based on a urinary protein level $<1.0 \mathrm{~g} /$ day [31]. The urinary protein level immediately after LDL-A and the rates of improvement of urinary protein, serum albumin, serum creatinine, eGFR, and TC and LDL-C after the LDL-A treatment session significantly affected the outcome.

In most studies, LDL-A was significantly associated with reduction of TC and LDL-C, and remission of NS in FGS or refractory NS patients. However, renal outcome due to LDL-A may depend on selectivity of proteinuria, duration of nephrotic 

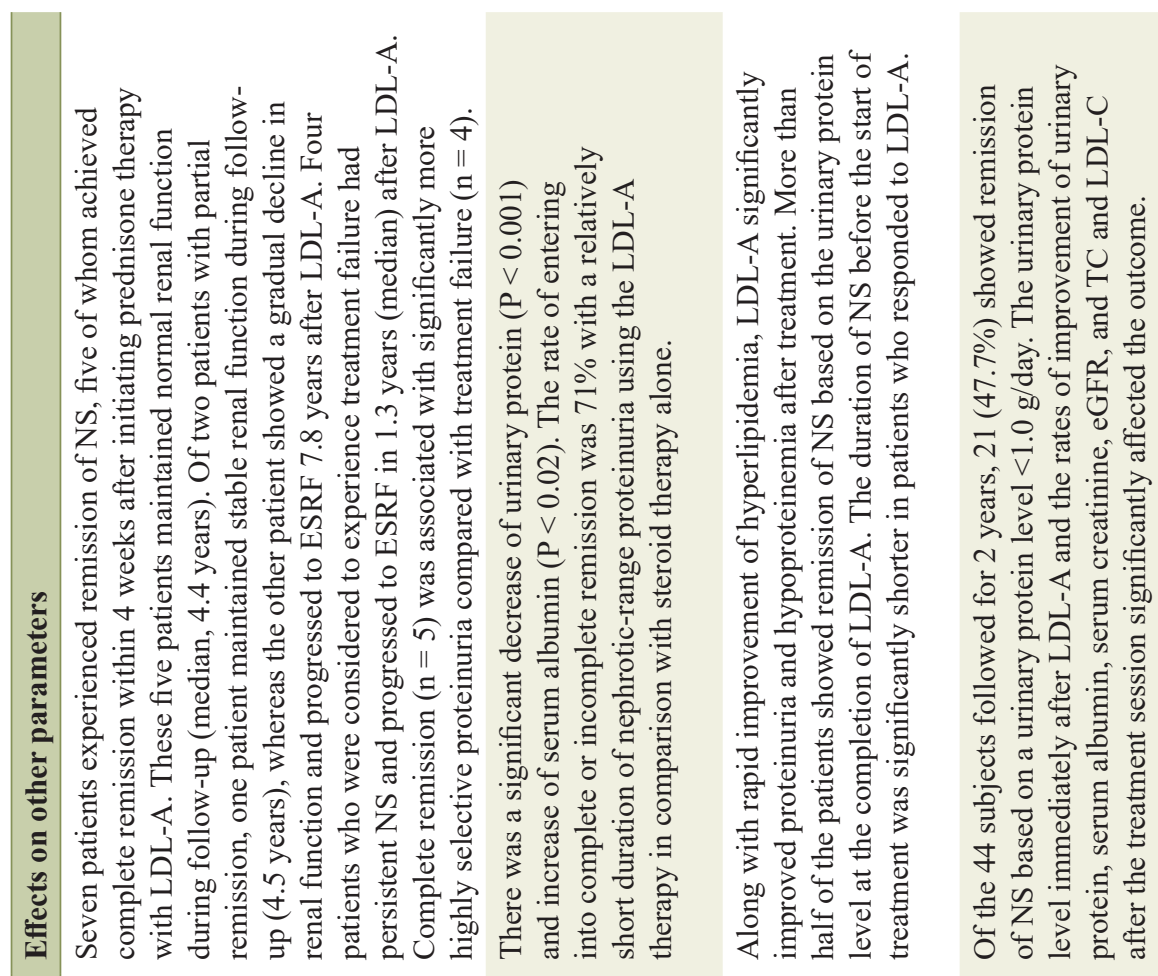

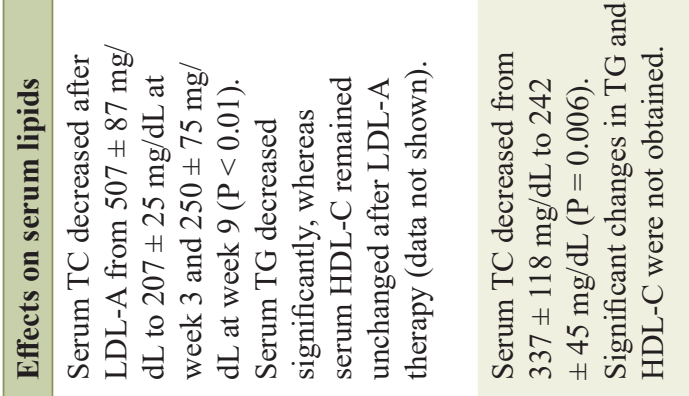

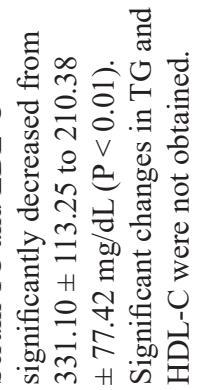
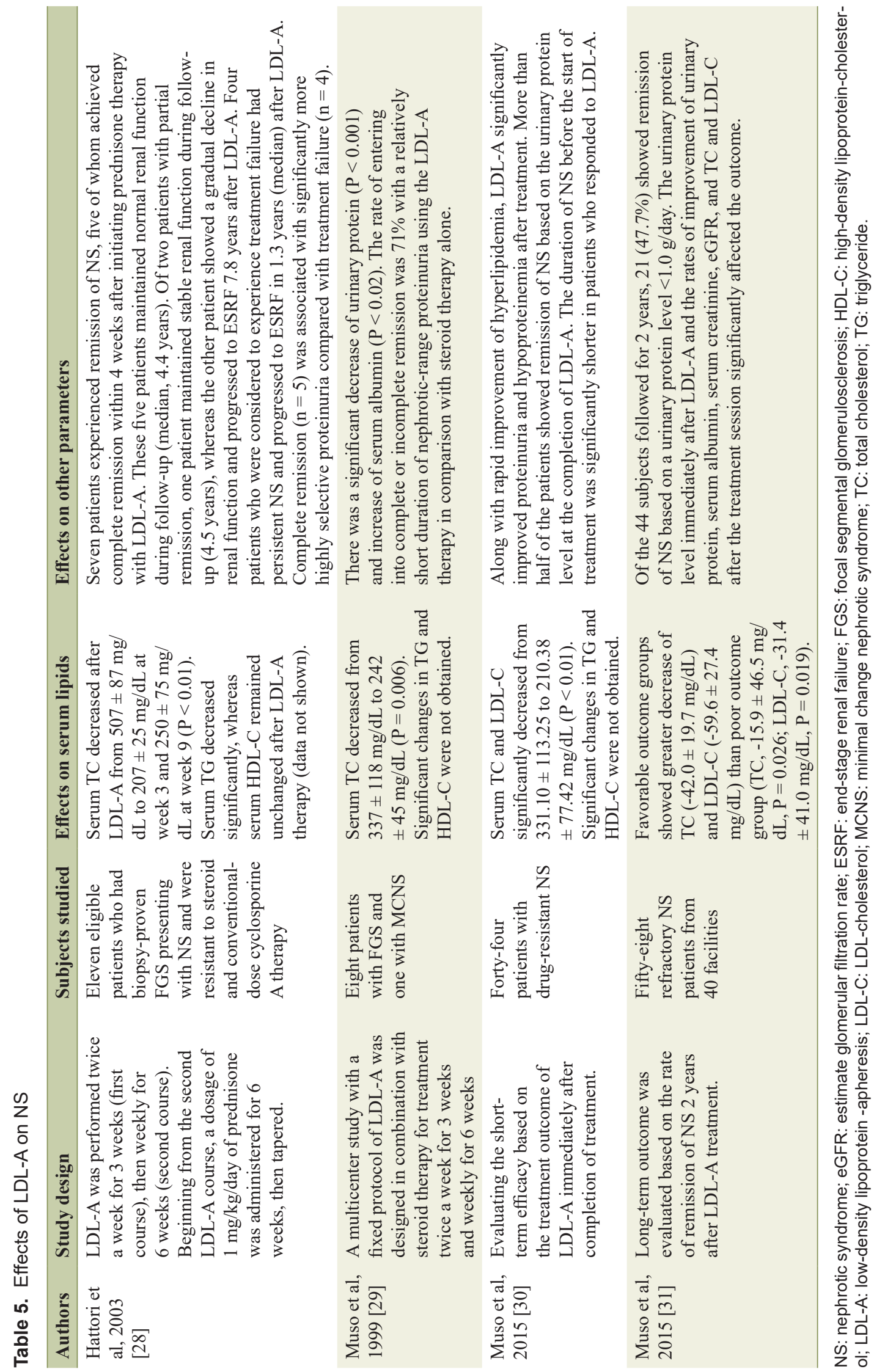
state and reactivity of lipids and urinary protein after LDL-A.

\section{PCSK9 inhibitors}

PCSK9 plays a crucial role in the regulation of cholesterol homeostasis and has thus gained considerable attention in the context of lipid-lowering strategies. Patients with NS showed a decrease in plasma cholesterol and plasma PCSK9 on remission of their disease $(\mathrm{P}<0.05, \mathrm{n}=47$ - 50) [32]. Podocyte damage was reported to trigger marked inductions in plasma PCSK9, and knockout of Pcsk9 ameliorates dyslipidemia in a mouse model of NS [32]. PCSK9 inhibitors may be beneficial in patients with NS. Large-scale trials which investigate the effects of PCSK9 inhibitors in NS patients is desired in the future.

\section{Conclusions}

Although dyslipidemia may be associated with atherosclerosis in NS, other factors such as age, duration of disease, number of relapses and blood pressure are also crucial determinants of atherosclerosis. The disease-specific risk of thromboembolism was different across the histological groups. One cohort study suggested that persons with NS are at increased risk of CHD. Among various interventions for NS, statin is relatively safe and effective for hyperlipidemia due to NS, but, it does not show sufficient evidence for renal outcomes. Although PCSK9 inhibitors is promising therapeutic option for NS, large-scale trials are needed to elucidate such effect.

\section{Acknowledgments}

None to declare.

\section{Financial Disclosure}

Author has no financial disclosures to report.

\section{Conflict of Interest}

The author declares that he has no conflict of interest concerning this article.

\section{Author Contributions}

H.Y. collected literatures and wrote and approved the final paper.

\section{Data Availability}

The data supporting the findings of this study are available from the corresponding author upon reasonable request.

\section{References}

1. Greenbaum LA, Benndorf R, Smoyer WE. Childhood nephrotic syndrome - current and future therapies. Nat Rev Nephrol. 2012;8(8):445-458.

2. Ponticelli C, Villa M, Banfi G, Cesana B, Pozzi C, Pani A, Passerini $\mathrm{P}$, et al. Can prolonged treatment improve the prognosis in adults with focal segmental glomerulosclerosis? Am J Kidney Dis. 1999;34(4):618-625.

3. MacHardy N, Miles PV, Massengill SF, Smoyer WE, Mahan JD, Greenbaum L, Massie S, et al. Management patterns of childhood-onset nephrotic syndrome. Pediatr Nephrol. 2009;24(11):2193-2201.

4. Agrawal S, Zaritsky JJ, Fornoni A, Smoyer WE. Dyslipidaemia in nephrotic syndrome: mechanisms and treatment. Nat Rev Nephrol. 2018;14(1):57-70.

5. Mehta A, Mishra S, Ahmad K, Tiwari HC, Singh V, Singh A. Carotid intima media thickness in children with nephrotic syndrome: an observational case control study. Sudan J Paediatr. 2019;19(2):110-116.

6. Alves C, Pinho JF, Dos Santos LM, Magalhaes G, da Silva JM, Fontes FL, Caligiorne SM, et al. Augmentation index, a predictor of cardiovascular events, is increased in children and adolescents with primary nephrotic syndrome. Pediatr Nephrol. 2020;35(5):815-827.

7. Kerlin BA, Ayoob R, Smoyer WE. Epidemiology and pathophysiology of nephrotic syndrome-associated thromboembolic disease. Clin JAm Soc Nephrol. 2012;7(3):513-520.

8. Loscalzo J. Venous thrombosis in the nephrotic syndrome. N Engl J Med. 2013;368(10):956-958.

9. Ordonez JD, Hiatt RA, Killebrew EJ, Fireman BH. The increased risk of coronary heart disease associated with nephrotic syndrome. Kidney Int. 1993;44(3):638-642.

10. Zou PM, Li H, Cai JF, Chen ZJ, Li C, Xu P, Li MX, et al. A Cohort Study of Incidences and Risk Factors for Thromboembolic Events in Patients with Idiopathic Membranous Nephropathy. Chin Med Sci J. 2018;33(2):91-99.

11. Harza M, Ismail G, Mitroi G, Gherghiceanu M, Preda A, Mircescu G, Sinescu I. Histological diagnosis and risk of renal vein thrombosis, and other thrombotic complications in primitive nephrotic syndrome. Rom J Morphol Embryol. 2013;54(3):555-560.

12. van den Brand JA, van Dijk PR, Hofstra JM, Wetzels JF. Long-term outcomes in idiopathic membranous nephropathy using a restrictive treatment strategy. J Am Soc Nephrol. 2014;25(1):150-158.

13. Mahmoodi BK, ten Kate MK, Waanders F, Veeger NJ, Brouwer JL, Vogt L, Navis G, et al. High absolute risks and predictors of venous and arterial thromboembolic events in patients with nephrotic syndrome: results from a large retrospective cohort study. Circulation. 2008;117(2):224230.

14. Lechner BL, Bockenhauer D, Iragorri S, Kennedy TL, Siegel NJ. The risk of cardiovascular disease in adults who have had childhood nephrotic syndrome. Pediatr 
Nephrol. 2004;19(7):744-748.

15. D'Amico G, Gentile MG, Manna G, Fellin G, Ciceri R, Cofano F, Petrini C, et al. Effect of vegetarian soy diet on hyperlipidaemia in nephrotic syndrome. Lancet. 1992;339(8802):1131-1134.

16. Gentile MG, Fellin G, Cofano F, Delle Fave A, Manna G, Ciceri R, Petrini C, et al. Treatment of proteinuric patients with a vegetarian soy diet and fish oil. Clin Nephrol. 1993;40(6):315-320.

17. Bell S, Cooney J, Packard CJ, Caslake MJ, Deighan CJ. The effect of omega-3 fatty acids on the atherogenic lipoprotein phenotype in patients with nephrotic range proteinuria. Clin Nephrol. 2012;77(6):445-453.

18. Rabelink AJ, Hene RJ, Erkelens DW, Joles JA, Koomans HA. Effects of simvastatin and cholestyramine on lipoprotein profile in hyperlipidaemia of nephrotic syndrome. Lancet. 1988;2(8624):1335-1338.

19. Gheith OA, Sobh MA, Mohamed Kel S, El-Baz MA, ElHusseini F, Gazarin SS, Ahmed HA, et al. Impact of treatment of dyslipidemia on renal function, fat deposits and scarring in patients with persistent nephrotic syndrome. Nephron. 2002;91(4):612-619.

20. Thomas ME, Harris KP, Ramaswamy C, Hattersley JM, Wheeler DC, Varghese Z, Williams JD, et al. Simvastatin therapy for hypercholesterolemic patients with nephrotic syndrome or significant proteinuria. Kidney Int. 1993;44(5):1124-1129.

21. Olbricht CJ, Wanner C, Thiery J, Basten A. Simvastatin in nephrotic syndrome. Simvastatin in Nephrotic Syndrome Study Group. Kidney Int Suppl. 1999;71:S113-116.

22. Coleman JE, Watson AR. Hyperlipidaemia, diet and simvastatin therapy in steroid-resistant nephrotic syndrome of childhood. Pediatr Nephrol. 1996;10(2):171-174.

23. Sanjad SA, al-Abbad A, al-Shorafa S. Management of hyperlipidemia in children with refractory nephrotic syndrome: the effect of statin therapy. J Pediatr. 1997;130(3):470-474.

24. Hari P, Khandelwal P, Satpathy A, Hari S, Thergaonkar R, Lakshmy R, Sinha A, et al. Effect of atorvastatin on dyslipidemia and carotid intima-media thickness in children with refractory nephrotic syndrome: a randomized controlled trial. Pediatr Nephrol. 2018;33(12):2299-2309.

25. Groggel GC, Cheung AK, Ellis-Benigni K, Wilson DE. Treatment of nephrotic hyperlipoproteinemia with gemfibrozil. Kidney Int. 1989;36(2):266-271.

26. Buyukcelik M, Anarat A, Bayazit AK, Noyan A, Ozel A, Anarat R, Aydingulu H, et al. The effects of gemfibrozil on hyperlipidemia in children with persistent nephrotic syndrome. Turk J Pediatr. 2002;44(1):40-44.

27. Yamashita S, Masuda D, Matsuzawa Y. Pemafibrate, a new selective PPARalpha modulator: drug concept and its clinical applications for dyslipidemia and metabolic diseases. Curr Atheroscler Rep. 2020;22(1):5.

28. Hattori M, Chikamoto H, Akioka Y, Nakakura H, Ogino D, Matsunaga A, Fukazawa A, et al. A combined lowdensity lipoprotein apheresis and prednisone therapy for steroid-resistant primary focal segmental glomerulosclerosis in children. Am J Kidney Dis. 2003;42(6):11211130.

29. Muso E, Mune M, Fujii Y, Imai E, Ueda N, Hatta K, Imada $\mathrm{A}$, et al. Low density lipoprotein apheresis therapy for steroid-resistant nephrotic syndrome. Kansai-FGSApheresis Treatment (K-FLAT) Study Group. Kidney Int Suppl. 1999;71:S122-125.

30. Muso E, Mune M, Hirano T, Hattori M, Kimura K, Watanabe T, Yokoyama H, et al. Immediate therapeutic efficacy of low-density lipoprotein apheresis for drug-resistant nephrotic syndrome: evidence from the short-term results from the POLARIS Study. Clin Exp Nephrol. 2015;19(3):379-386.

31. Muso E, Mune M, Hirano T, Hattori M, Kimura K, Watanabe T, Yokoyama $\mathrm{H}$, et al. A prospective observational survey on the long-term effect of LDL apheresis on drug-resistant nephrotic syndrome. Nephron Extra. 2015;5(2):58-66.

32. Haas ME, Levenson AE, Sun X, Liao WH, Rutkowski JM, de Ferranti SD, Schumacher VA, et al. The role of proprotein convertase subtilisin/Kexin type 9 in nephrotic syndrome-associated hypercholesterolemia. Circulation. 2016;134(1):61-72. 